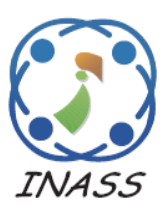

\title{
Integration of Indoor Localization System using Wi-Fi Fingerprint, Bluetooth Low Energy Beacon and Pedometer Based on Android Application Platform
}

\author{
Khamla NonAlinsavath ${ }^{1,2 *}$ \\ Lukito Edi Nugroho ${ }^{1}$ \\ Kazuhiko Hamamoto ${ }^{2}$ \\ Widyawan Widyawan ${ }^{1}$ \\ ${ }^{I}$ Department of Electrical Engineering and Information Technology, Faculty of Engineering, \\ Universitas Gadjah Mada Yogyakarta, Indonesia \\ ${ }^{2}$ Department of Information Media Technology, School of Information and Telecommunication Engineering, \\ Tokai University, Tokyo, Japan \\ * Corresponding author’s Email: khamla@mail.ugm.ac.id
}

\begin{abstract}
Location-based service has become an important element in indoor positioning with increasing precision. Indoor technologies help with user navigation and positioning in specific locations. Many location-based services and applications have been developed to improve indoor positioning, especially to deal with the complexity of location awareness systems. This study proposes the use of Wi-Fi Fingerprint (Wi-Fi), pedometer and Bluetooth Low Energy 4.0 (BLE) beacon to measure and pinpoint user's indoor locations using received signal strength-based indoor localization. The system tracks, directs and guides user's indoor movements using an android application and assists first-time visitors to find a particular location. The networks are comparable and can be detected using smartphones. The phones will detect the strongest signal from the available networks. Wi-Fi, pedometer and BLE 4.0 beacon were able to identify the direction and location in indoor environments and assist user to find their specific destination easily. The map provided by the system based on position information has an accuracy of $91.5 \%$ at the third floor and $97.1 \%$ at the second floor, and the signals have seamlessly worked together in integrated fashion when user is in indoor environment. The system is also able to highlight pathway according to user's current location and destination, in addition to being fast responding, secure and robust. The application enables seamless service in real-time operation and provides multiple pathways, guidance and highlighted map for indoor localization. The display of distance between the user and the destination as the user near the target destination is an extremely helpful feature in the application.
\end{abstract}

Keywords: Indoor positioning, Location awareness, Navigation system, Location tracking.

\section{Introduction}

Location information [1] used for navigation and tracking has improved tremendously in terms of accuracy and availability for indoor uses and accessing nearby locations. A positioning system is vital to users, as it allows them to locate positions especially indoor locations from the outside. Indoor positioning system [2] is a location technology that helps users by providing directions and indoor locations using applications installed on smart devices. These systems are able to measure, locate, and display specific coverage areas, and provide information in terms of maps to specific destinations or locations [3]. Global Positioning System (GPS) [4] performs well outdoors, but it does not work well indoors such as in buildings, due to weak signal availability. There are other location technologies that can be used indoors such as Wi-Fi [5], BLE [6] and RFID (Radio Frequency Identification) [7], pedometer [8], ZigBee [9] and so on. These technologies can be easily integrated allowing for connectivity and communication between users, devices, and systems to assist navigation.

Indoor localization technologies [10] can be categorized into the following: proximity, geometrical, measurement and fingerprinting 
techniques. Most use radio signal strength (RSS) to estimate relative locations especially indoors and are efficient due to being small, cheap and easy to setup, rendering low power consumption. Meanwhile, BLE beacon has also been commonly used indoors, which works by emitting low energy signal via smartphones. Indoor positioning system holds values both commercially and academically as it supports daily lives, making it more convenient to navigate and saves time and resources to get to various places like university campuses, shopping centers, indoor stadiums, and so on. Thus, there is constant need to improve the technology and performance of indoor positioning systems to simplify, enhance, and improve location awareness indoors.

Many researches have discussed the use of Wi-Fi and BLE beacon for relevant application related to indoor positioning services. Wi-Fi works indoors when its signal is available and accessible. BLE beacon is a high-quality alternative that works well with other indoor positioning technologies. It is energy-efficient, provides quick respond and works effectively in spaces like footpaths, rooms and multifloor buildings.

However, there are some challenges in using the system such as position accuracy, indoor map availability, location privacy, service continuity from outdoor to indoor area, limitation in signal in available indoor, and practicality since the system relies on various components such as device, technologies, and environment. Uncertainty in the signal around the building or indoor location adds to the challenge when user needs to find specific location, and available signal sometimes does not cover all area. It is difficult to design a system where user can appoint another person(s) to provide accurate directions without assistance from others, or in the absence of people in a complex location. Furthermore, the challenge in developing indoor localization system has been a huge obstacle for indoor application that relies on availability and accuracy of indoor positioning service, as lack of accurate positioning is the key issue for tracking system in indoor environment. The application needs to be able to localize user accurately and seamlessly, offering reliable, inexpensive indoor positioning and providing location information that is fully attached to the environment and proposed system. [11]. This research proposes an indoor positioning system to provide, detect, localize target positions accurately, and plan routes for user to travel from their current location to a desired location using $\mathrm{Wi}-\mathrm{Fi}$, pedometer and BLE 4.0 beacon. The system will track peoples' location, monitors the environment, and provides real-time support and uninterrupted service. Indoor
Technologies work together to overcome each other disadvantages: Wi-Fi that consumes more energy than BLE; BLE that is less accurate compared to WiFi. By obtaining better localization and improving positioning algorithms, the proposed system will have better performance as compared to mechanisms proposed in other research works as outlined in Table 1.

This research paper is divided into the following: section 2 presents the related works on indoor localization; section 3 discusses the system implementation and experimental setup for an indoor positioning system; section 4 analyses the results of the experiment involving the system; section 5 provides a conclusion.

\section{Related Works}

Indoor positioning systems $[12,13]$ have been developed and researched extensively. They have been used to navigate indoors in places like hospitals, shopping malls, campus buildings, and warehouses. They have been commercialized and further researches are ongoing to improve the products on offer. [14] proposed an android application which enables efficient indoor navigation that tracks peoples' exact location inside a large building by using built-in sensors in smartphones like magnetometers, gyroscope, and accelerometer. The application can run without any infrastructural facilities like Wi-Fi access points, BLE beacon dongles, RFID readers or sensor chips. The application was tested successfully in a selected indoor location within the institute, and it generated accurate results in different climatic conditions. The application is lighter, more user-friendly and consumes less memory and CPU time than the existing application. P.K Binu [15] presented a system to determine vehicle location indoors by combining location fingerprinting using BLE beacon and relative positioning using vehicle sensor data via controller area network (CAN). The two components used a combination of Kalman and particle filter incorporated in the building's layout. The proposed system was tested in real-life situation, where a test vehicle was driven into a large parking garage along a predefined route through the building. The system showed better performance with the map information acting as an additional sensor input to overcome existing constraints. However, authors found out several obstacles during the experiment; it was challenging to generate accurate value for each sampling point due to continuous fluctuations in magnetic field, and in the event that the user's walking patten is different from the trainer's. 
Table 1. Benchmarking between the proposed system and existing inventions

\begin{tabular}{|l|l|l|l|l|}
\hline \multicolumn{1}{|c|}{ Name } & \multicolumn{1}{c|}{ Accuracy } & \multicolumn{1}{c|}{ Cost } & \multicolumn{1}{c|}{ Coverage } & \multicolumn{1}{c|}{ Benefits and drawbacks } \\
\hline $\begin{array}{l}\text { Active Badge (Infrared } \\
\text { Bases) [19] }\end{array}$ & $1 \mathrm{~m}-5 \mathrm{~m}$ & Low & Poor indoor & $\begin{array}{l}\text { Short-range detection; no clearance of } \\
\text { multipath. }\end{array}$ \\
\hline $\begin{array}{l}\text { Active Bats (Ultrasound) } \\
{[20]}\end{array}$ & $3 \mathrm{~m}-5 \mathrm{~m}$ & High & Medium indoor & Sensitive to environment. \\
\hline $\begin{array}{l}\text { Mall of America (Wi-Fi) } \\
{[21]}\end{array}$ & $1 \mathrm{~m}-5 \mathrm{~m}$ & Low & Good indoor & $\begin{array}{l}\text { Clear multipath; high processing } \\
\text { power; limitation in mobility. }\end{array}$ \\
\hline $\begin{array}{l}\text { Proposed System (Wi-Fi, } \\
\text { BLE beacon, pedometer) }\end{array}$ & $1 \mathrm{~m}-5 \mathrm{~m}$ & Low & $\begin{array}{l}\text { Good indoor and } \\
\text { outdoor }\end{array}$ & $\begin{array}{l}\text { Infrastructure available everywhere; } \\
\text { clear multipath; high processing } \\
\text { power and speed; multiple available } \\
\text { signals integration. }\end{array}$ \\
\hline
\end{tabular}

W. Jiang [16] presented a seamless indoor-outdoor navigation system based on GNSS (Global Navigation Satellite System), INS (Inertial Navigation System) and terrestrial ranging techniques that are able to support accurate indooroutdoor positioning. The system performed well both indoors and outdoors since the results show that GNSS, Locata, and INS were all able to provide navigation information. Fusing the observations from the three sensors seems to be the best solution in terms of reliability and accuracy. However, the positioning accuracy in the system can be compromised by the severe multipath disturbance in metal-clad warehouse, and the difference in operation between indoor and outdoor environment whereby, the outdoor is still less stable than indoor in real-life environment.

In [17], the author presented an android platform to implement step detection in order to assist in collecting radio fingerprints based on quick radio fingerprint collection (QRFC) algorithm, divided into two algorithms called moving sampling (MS) and stepped moving sampling (SMS). The findings can aid researchers studying the application of smartphone android platforms for indoor positioning application, and ways to collect appropriate signals through simple methods, with reduced errors and improved accuracy. An experiment to collect signals was done along a hallway of 54 meters in length and the signals took between 150 to 300 seconds for MS and SMS, respectively. The strength of signals obtained in the test reflected real-life situations more accurately than other systems as the user held the smartphones by hand during movement and positioning. Safei [18] proposed a trilateration technique to determine user's position indoors based on the strength of Wi-Fi signal with IEEE $802.11 \mathrm{~g}$ from access points indoors, and proposed a formula to analyze the distance between user and each access point. The proposed formula and trilateration technique can be used to determine and calculate user's position indoors. However, to determine the position of user indoor based on Wi-Fi signal strength, the system need to classify a clear solution for position detection.

\section{Methodology and Experiment Setup}

\subsection{System implementation}

An indoor positioning system was set up using Wi-Fi and BLE 4.0 beacon to identify and guide user accurately to a specific location indoors from the outside. BLE beacon is a small device that consumes very low energy, able to measure indoor environment precisely and communicate with other devices. The proposed system used BLE beacon and Wi-Fi to map the user's route between the source and destination. The system would show the availabie signals, where the user can connect to the strongest signal in the application. The application would collect all information to assist with positioning and direct the user to destination.

Android platform acts as a user interface in the proposed system, utilized by other smartphone applications as a location finder and to specify positions by communicating and transferring information between the user and the system.

BLE beacon was used to transmit signals if there was no Wi-Fi signal available in the building. Sometimes it could be impossible to collect fingerprints as some area may not be connected to a Wi-Fi. When setting up the BLE it is important to consider the range of the network and distance between devices. If the current location can detect Wi-Fi signals, then the system would choose Wi-Fi mode automatically for the user. BLE is suitable to be used indoors since it is cheap, small, and consumes less energy. Devices also work well on an android platform, and do not require many components and can support both Wi-Fi and BLE beacon to obtain directions and specific locations. 

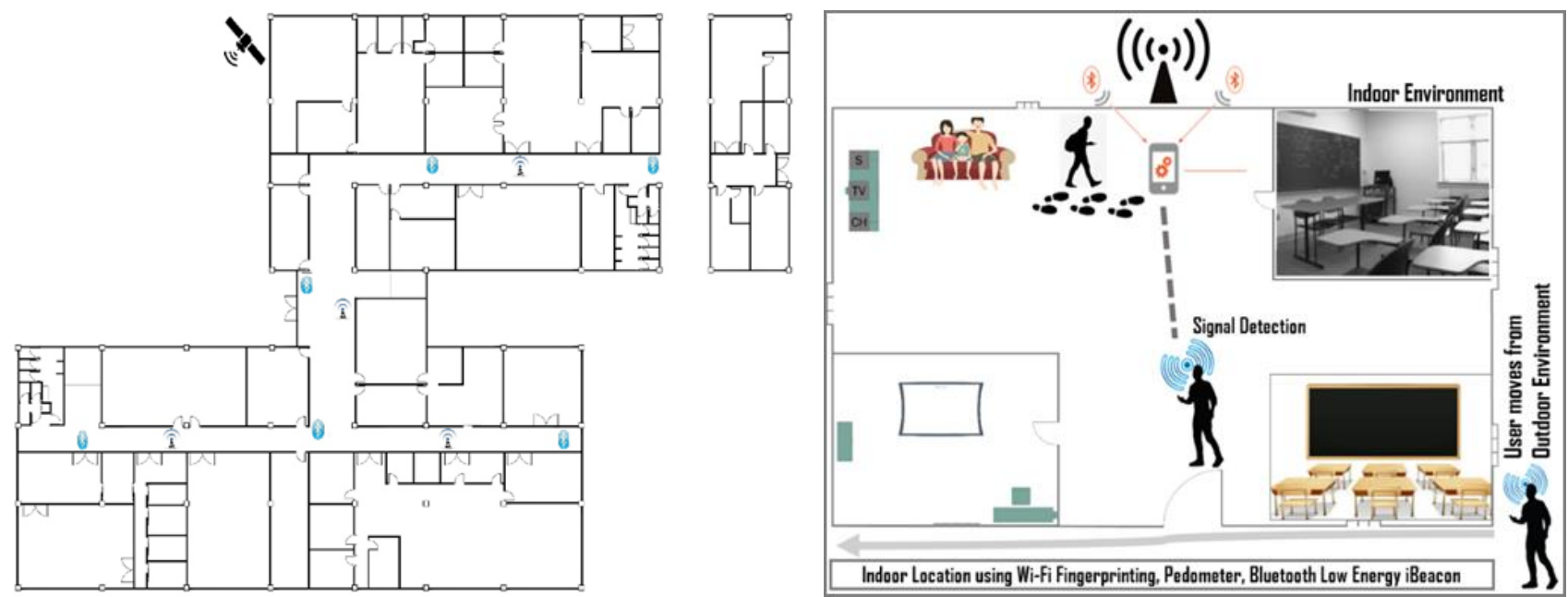

Figure. 1 Floor plan of the first floor in JTETI Building (left) and system design for indoor localization using Wi-Fi, pedometer, and BLE beacon (right)

In order to evaluate the efficiency of the system in an indoor environment, the study combined Wi-Fi, pedometer and BLE beacon technologies. Experiment (Fig. 1) was designed by placing Wi-Fi access points and BLE beacon together to collect route information from various locations to other destinations without hindrances. Pedometer technology was used to count user's steps and specifiy the distance between current location and destination, as well as to measure the accuracy of indoor direction when user moves from overlapped area to a specific indoor position. The study measured signal strength by collecting fingerprint signals from Wi-Fi access points and BLE beacon transmitters using a smartphone (Samsung Galaxy S7 Edge) to communicate, direct user and process information. The smartphone detected signals from all available networks and the system would decide to latch on to the strongest signal, and used the signal to direct user to destination.

Smartphone equipped with Wi-Fi, pedometer and BLE beacon modes was used to collect information on the strength of received signal between two sites before sending them to the system. The signals were continuously scanned at the base stations of the respective technologies to obtain the strongest signal that can assist the user in moving to target location.

The system used an indoor localization map to map out the building interior for better accuracy while tagging the route for user i.e. the length of pathway, floors inside the building etc. The system took few seconds to search for the best signals among the available networks (Fig. 2). The BLE beacon and Wi-Fi data provided various information like user's position indoors, their estimated position and the distance between Wi-Fi and BLE beacon.

\subsection{Experiment setup}

Numerous BLE beacon and Wi-Fi access points were set up inside the building of Electrical Engineering and Information Technology, Faculty of Engineering, Universitas Gadjah Mada (called as JTETI Building as shown in Fig. 1 (left)). The proposed location is a three-floor building with different architectural and interior design. BLE beacon were placed every 15 meters from the entrance and Wi-Fi access points were placed every 50 meters to ensure full indoor coverage. The participating user used a Samsung Galaxy S7 Edge smartphone, equipped with detector of various signals such Wi-Fi, BLE beacon, pedometer and others.

We estimated the user's current position and the attached devices around the building using Euclidean metric, which measures the vector of fingerprint $x=$ $\left(x_{1}, x_{2}, \ldots, x_{n}\right)$ at the $\mathrm{n}$ space from other point $y=\left(y_{l}\right.$, $\left.y_{2}, \ldots y_{n}\right)$. The distance $(d)$ between two different points from $x$ to y position was calculated as follows:

$$
d(x, y)=\sqrt{\left(y_{1}-x_{1}\right)^{2}+\left(y_{2}-x_{2}\right)^{2}}
$$

We implemented the system by combining three networking technologies namely Wi-Fi, BLE beacon and pedometer in order to provide available alternative when user missed the signals from one of the networks. The signal was classified and calculated as follows: 


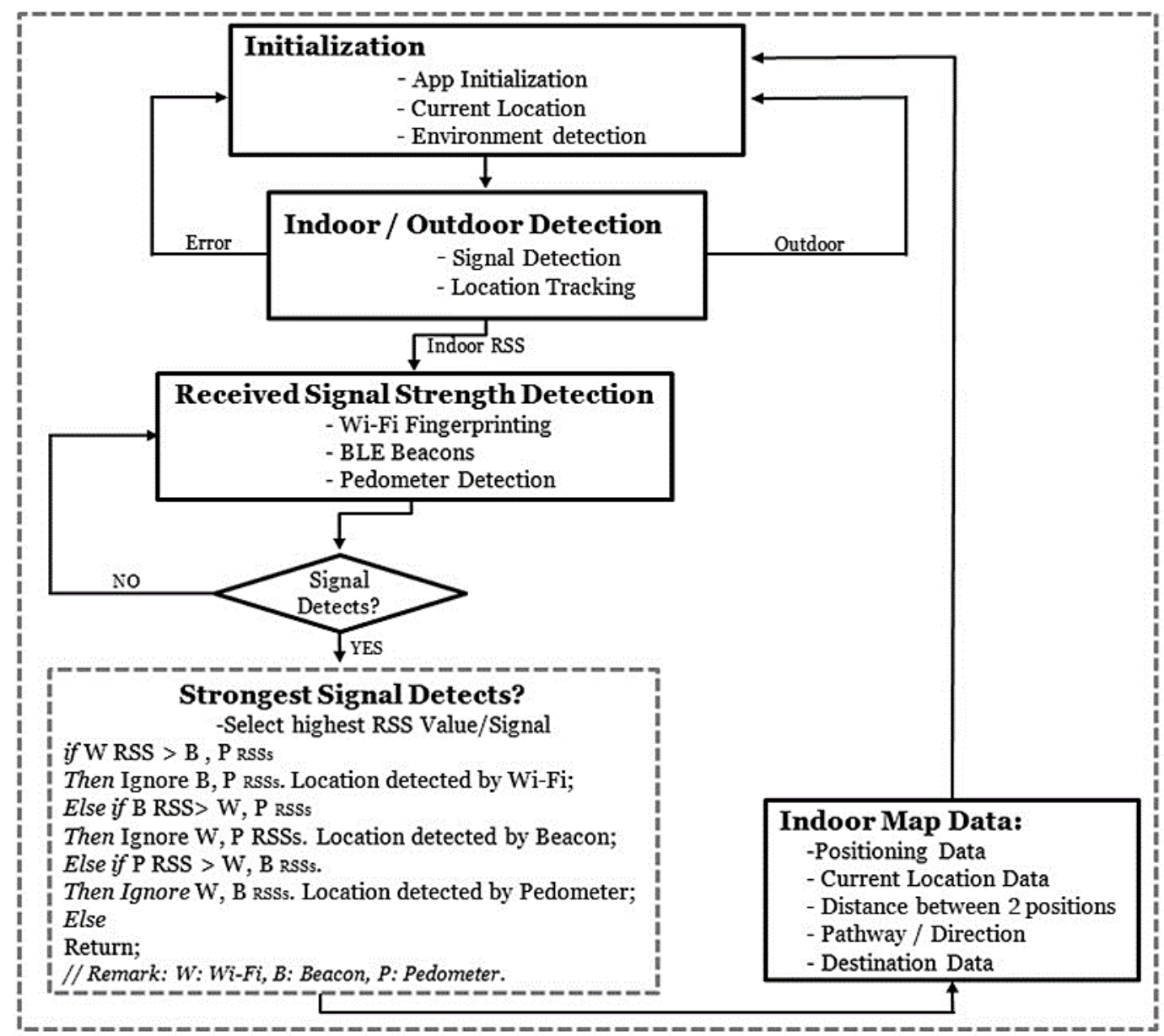

Figure. 2 Flowchart of the proposed system

$$
\left\{\begin{array}{l}
d_{1}=\sqrt{\left(y_{w 1}-x_{w 1}\right)^{2}+\left(y_{w 2}-x_{w 2}\right)^{2}} \\
d_{2}=\sqrt{\left(y_{b 1}-x_{b 1}\right)^{2}+\left(y_{b 2}-x_{b 2}\right)^{2}} \\
d_{3}=\sqrt{\left(y_{p 1}-x_{p 1}\right)^{2}+\left(y_{p 2}-x_{p 2}\right)^{2}}
\end{array}\right.
$$

Received signal strength expressed as power at a particular distance, $d\left(d_{1}, d_{2}, d_{3}, \ldots d_{n}\right)$ from one point to another throughout different range of points and environments was calculated using equation 2: $w$ represents the strength of received $\mathrm{Wi}-\mathrm{Fi}$ signal between two different positions, point $x_{w}$ to point $y_{w}$; $B$ represents the strength of received BLE beacon signal between point $x_{b}$ to point $y_{b} ; p$ represents the strength of pedometer signal from point $x_{p}$ to point $y_{p}$. Combination of the three localization technologies can provide user with multiple alternatives to maintain connection with the system in the event of access failure to one of the localization technologies, where user can swiftly switch to another localization mode with stronger signal. The combined indoor technologies could enhance the usability and accuracy of user's indoor positioning.

\subsection{Test specification and condition classification}

In order to make sure the system and application were successfully implemented, we designed application testing and conditions (Table 1) to analyze the environment and input, which include components of decision and condition coverage, multiple conditions and black box test. Test design is a process of providing input to the system and checking on how the system processes the input to generate output when the system was implemented in real environment and assigned indoor conditions, where user moved from outdoor to indoor. This test helps to uncover defects at structural level and complexity in application operation. The tests was carried out from bottom to the top, in addition to functional layer to ensure system adaptation to environmental changes and recognition of building information such as rooms, floors, pathway, reference points and level of the floor based on coordinates of point. 
Table 2. Example of condition testing and specification

\begin{tabular}{|c|c|c|c|}
\hline No. & Input & Expected & Actual \\
\hline 1 & $\begin{array}{l}\text { 1. Store Rooms }==\text { Store Floor } \\
\text { 2. Enter }\end{array}$ & $\begin{array}{l}\text { Registration completed } \\
\text { successfully }\end{array}$ & $\begin{array}{l}\text { Registration completed } \\
\text { successfully }\end{array}$ \\
\hline 2 & $\begin{array}{l}\text { 1. Store Rooms }==\text { Store Floors } \& \& \\
\text { Enter }==\text { Register }\end{array}$ & $\begin{array}{l}\text { Saved rooms/floors into the } \\
\text { application }\end{array}$ & $\begin{array}{l}\text { Saved rooms/floors into the } \\
\text { application }\end{array}$ \\
\hline 3 & $\begin{array}{l}\text { 1. Store Rooms }==\text { Store Floors } \& \& \\
\text { Enter }==\text { Register } \\
\text { 2. Store Room }==\text { Store Floors }\end{array}$ & Registration is not completed & Registration was not completed \\
\hline 4 & $\begin{array}{l}\text { 1. Store map }==\text { Scan SDK result \&\& } \\
\text { Enter }\end{array}$ & $\begin{array}{l}\text { Registration completed } \\
\text { successfully }\end{array}$ & $\begin{array}{l}\text { Registration completed } \\
\text { successfully }\end{array}$ \\
\hline
\end{tabular}

Table 3. Test scenario at assigned location and reference points

Test Scenario No. 01

Purpose: To verify that user's location and entered points are matched with stored data in database

Cross-reference: Registration of pre-condition

Complete successful scenario

\begin{tabular}{|c|c|}
\hline User action & System response
\end{tabular}

1. User opens the application

1. System verifies email and password

2. User enters indoor application

3. Now user enters their rooms

2. System shows points/rooms on first fragment

4. Now user clicks registration

3. System shows complete map and provides pathway

4. Exact location of floors

5. User logs into the application

6. User sees first fragment (indoor tracking), by sliding user sees second fragment (map), and again by sliding user sees last fragment (graph data)

7. User searches for rooms on second fragment

Expected: Login successful and room location is given to the user Cross-reference: 02TC2

\begin{tabular}{|c|c|c|c|}
\hline Date & Result & Version & Run by \\
\hline $15^{\text {th }}$ Dec, 2019 & Pass & 1.2 & Admin \\
\hline
\end{tabular}

We assigned test scenario to verify that user's location and entered points are matched with stored data in database (Table 2). This is a process of giving input to the system and checking on how the system generates output in the application, and analyzing the reference points and database to help system and user meeting the system requirement specifications. The user action and system response were designed to be more effective than existing systems for large-scale application by improving indoor coverage using multiple indoor-based localization technologies namely Wi-Fi, BLE beacon and pedometer. Implementation of user interface and the system itself was successful as user was able to understand and recall the process easily, and use the system with ease when provided with complete information about the location and each task clearly.

\section{The experimental results and discussion}

The experiment was conducted using BLE beacon, pedometer on smartphone and $\mathrm{Wi}-\mathrm{Fi}$ access points deployed indoors. A smartphone was used to track user's location during a walk while carrying smartphone at assigned position. When user stepped on the floor, the system detected the direction and mapped out the path. The experiment involved calibration, path recording, location marking along each fixed point assigned by system developer. These were done to obtain high-quality results and improve accuracy of indoor positioning in different locations and situations.

After preliminary test classification, the key indoor environment was verified and matched with assigned condition based on real-time experiment. Location information of the building were added to the system to investigate current location available around the reference points; the system chose the most suitable indoor localization technique to implement in the application that renders low power consumption, operates in narrow space, and allows for continuity of service from outdoor to indoor.

The results show that the proposed system has properly captured user's current location. The system detects and tracks location via smartphone. The 

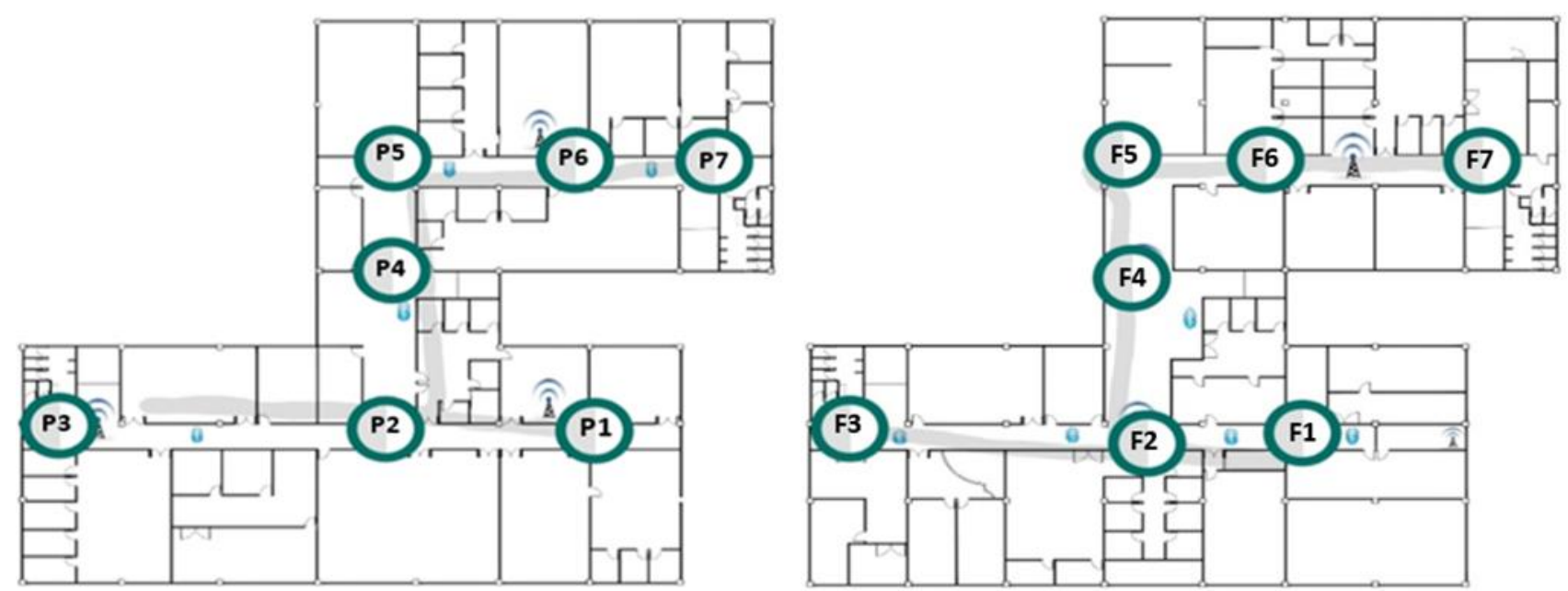

Figure. 3 Assigned position and the recorded path testing at the second floor (left) and third floor (right)

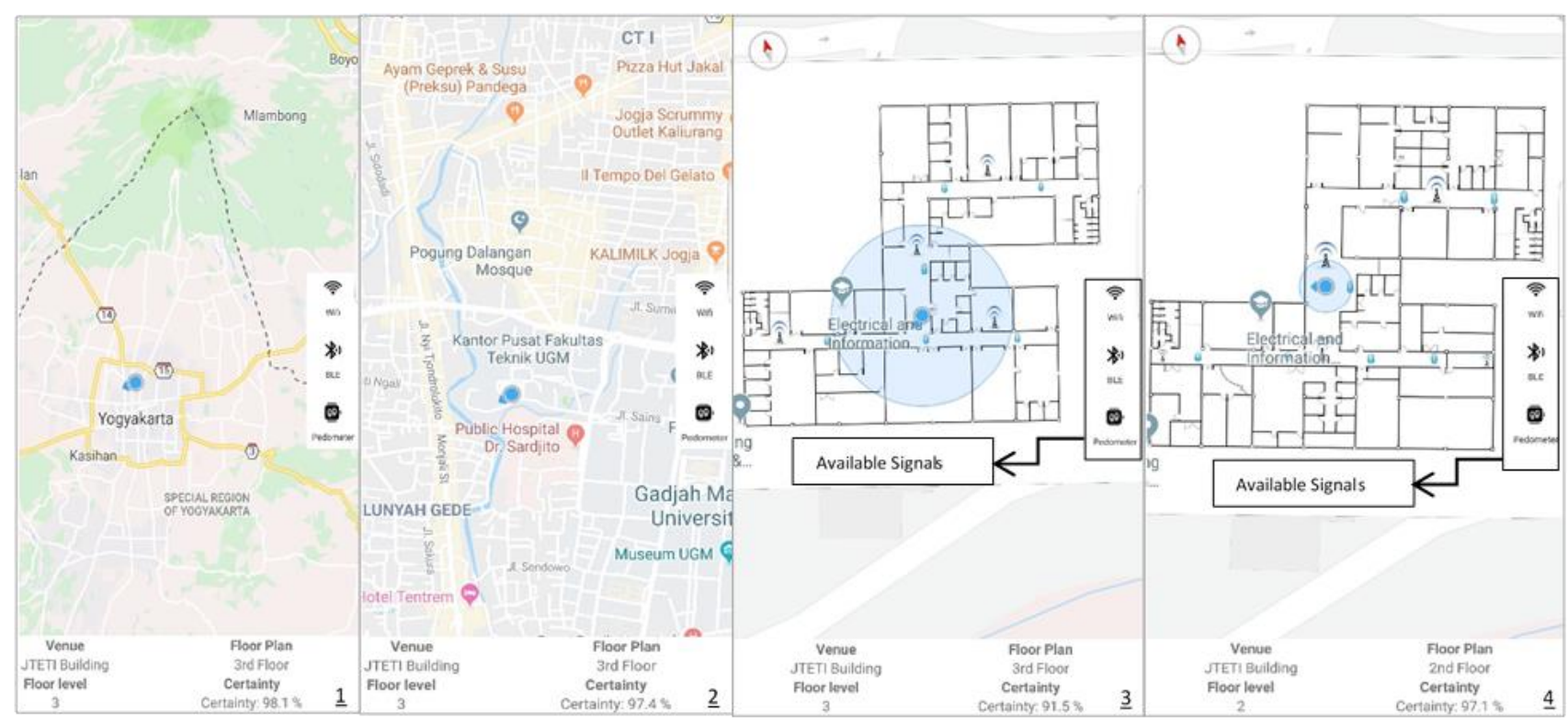

Figure. 4 Indoor location tracking and detection using available signals in real environment (1-4)

system collects location information such as distance between two locations, maps out the direction and user's movement from current location to the target destination. The system captures the real-time data reflecting the current situation. To support the location information, the system relies on the three indoor localization technologies, which signals are detectable using smartphone. The system detects the strongest signal among all available signals, and the smartphone chooses the strongest signal to guide user to the destination.

The study implemented the proposed system on the third floor of JTETI Building (Fig. 3). Several unknown locations were marked as " $F 1, F 2, F 3, F 4$, $F 5, F 6, F 7$ " at the second floor, and " $P 1, P 2, P 3, P 4$, $P 5, P 6, P 7$ " at the third floor as assigned fixed locations for the user to find and served as reference points. The indoor maps were firstly incorporated into the system with complete map information and floor plans to ensure that the application was able to fetch user's current location. As the user entered the mapped indoor space, the application triggered the indoor map and indicated user's navigation.

The reference points were assigned using real coordinates (longitude and latitude) collected by GPS navigation device. These values were recorded and inputted in the system using ArcGIS software. The experimental results (Fig. 3) show that the proposed system was able to track each reference point. It was also able to track the signals from the available networks and select the strongest signal to be used to measure distance and map out the path. The results (Fig. 4) show that the system could interact with user's device and available signals successfully. The user's device can track the location of the user moving between reference points and 
transmit signals correctly. The system supports and controls user's movement directly within a specific indoor setting. The system is able to detect various signals from different localization technologies and measure the efficiency based on information transmitted by the devices attached to the base of each location. The system collects information on the pathway as user moves along different points, which information is used as a reference to guide user with indoor navigation.

In real environment, when the user moved from outdoor to indoor space which pathway was already recorded using the proposed Android application, user's current location is continuously tracked using the signal from GPS, which can be switched to other available signals from indoor-based technologies namely Wi-Fi, BLE beacon or pedometer. Once the current location is detected, the application would start listening to the user's indoor position with complete information about the current positioning. The application provides measurement of each step using pedometer, and displays the indoor map to ensure that user enters the indoor space; extra features such as floor id, name and level of the floor provide complete indoor information for user.

Indoor location tracking via Android platform (Fig. 4 labelled as 1,2,3,4) starts by detecting three different signals, which provide location information and map of the area. First, the system detects user's current location; the system (application) obtains location information and an indoor map of the building as the user moves from outdoor to indoor space; incorporation of building plans into the system before system implementation allows the application to automatically zoom in when user positions themselves inside the building (indoor); the system detects the available signals in the area. Location information includes venue, floor plans, floor levels etc. (Fig. 4, right labelled as 3, 4). The map provided by the system based on positioning information has an accuracy of $91.5 \%$ at the third floor and $97.1 \%$ at the second floor. Accuracy depends on user's current location and the nearest position from assigned points and devices; certainty demonstrates how close the user is to the recorded path and matches the current longitude and latitude with the recorded points, which have been incorporated into the system prior to system implementation. Certainty indicates accuracy in detection of which floor the user is currently in, and the match between the recorded path and current position. The blue dot represents the current position of the user moving around the building, which changes depending on situation in indoor space.

The pathways of the building have specific reference points, which extend to all three floors. They have the same measurements to simplify positioning based on signal strength, set as per predetermined algorithms. However, errors may still occur due to variable signal strength in indoor space, which can be caused by obstacles inside the building such as thick walls and narrow spaces.

The system is able to detect each location accurately and plan the route to direct user from current location to destinations along the reference points.

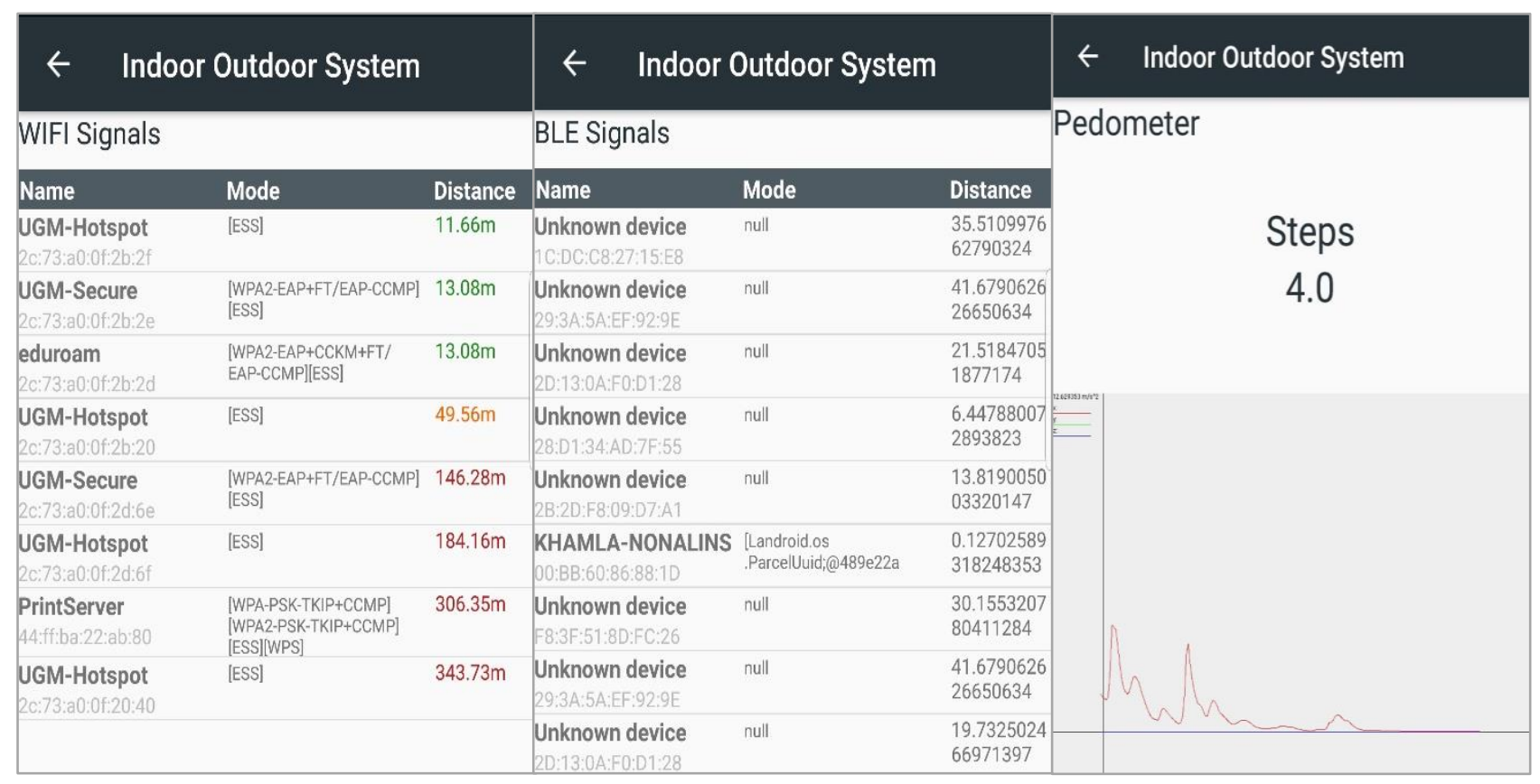

Figure. 3 Detection of signals from Wi-Fi (left), BLE beacon (middle) and pedometer (right) 
Fig. 5 shows the available signals detected by the system as the user moved around the building in search for the strongest signal. The system is able to detect available signals in the covered areas. Essentially, the system has performed as per its intended use - guiding and directing user indoors. Each interface works well and can be improved to facilitate the exchange of information among users directly from the system. The positioning component can be embedded into the infrastructure of the building. The results of the experiment are mostly in line with actual maps and reflect the existing environment. The experiment proved that existing technologies like Wi-Fi, BLE beacon and pedometer can be used in an indoor positioning system without compromising accuracy, while meeting most of the user requirement specification.

We find that this system has performed with high accuracy by demonstrating high level of certainty for indoor localization application. The system is proved reliable in guiding and directing user to destination as they move from outside to inside of the building. In terms of tracking, user's current location matched with the two indicators: recorded path along location coordinates and access points, which assured that the mapped out area is the same as in the recorded floor plan. One factor that continuously involved in the whole process is GPS location coordinates, which is incorporated into the system to detect the floor plan using available signals at a certain location. Indoor signals (BLE beacon, Wi-Fi and pedometer) appeared in the application as user entered the indoor space (Fig. 5). The system detects indoor signals to gain information regarding current location and position that match the recorded indoor maps, and notifies user as they enter or exit indoor space. In addition, positioning of indoor location has been significantly improved by providing location information such as distance between two different positions, direction map as user moves, and display of available signals. The accuracy has remarkably increased to $98.4 \%$, reflecting high level of certainty in indoor navigation. The distance is measured by how close the user is to the reference points and coordinates of a position; the system provides the length of pathway between the two different positions.

It can be seen in the results from another experiment that the positioning slightly deviated as user operated the application and current location was detected. Shown in Fig. 6, the Wi-Fi mode in the transition region outperformed other available signals at the location and at each reference point. However, there is risk in signal instability if the user moves too fast, or in narrow-spaced environment, or due to other

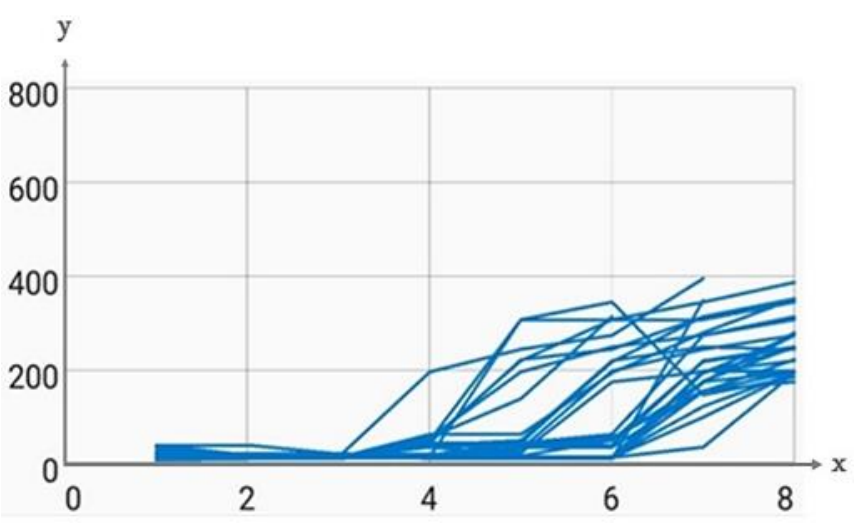

Figure. 4 Indoor experiments by using Wi-Fi signal

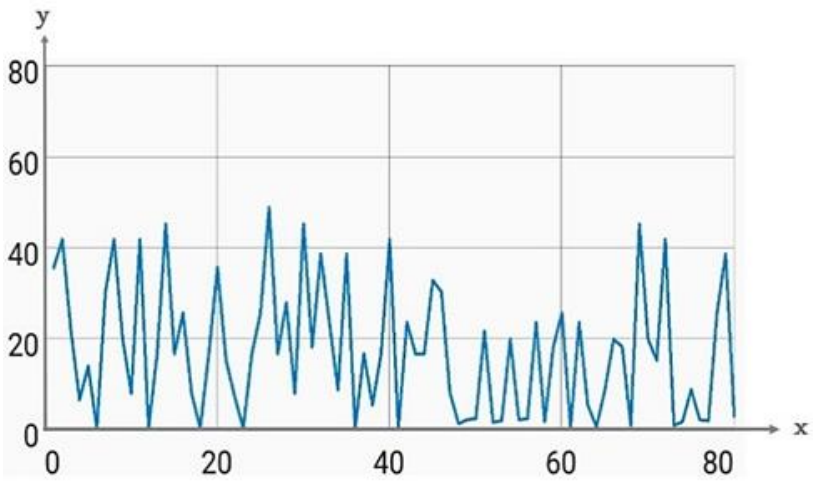

Figure. 5 Indoor experiments by using BLE beacon signal

unexpected factors. Wi-Fi signals are detectable from when the user starts to move, which can be observed in the graph representing signal detection, obtained as the system collects positioning data.

On the other hand, indoor positioning by using BLE beacon as shown in Fig. 7, resulted in indoor localization with high positioning accuracy. BLE beacon graph was generated as the user moved from one position to another to test the application. The graph plotted with time against the signal strength at current location illustrates the fluttering signal strength.

\section{Conclusion}

This paper discusses an indoor positioning system using Wi-Fi, BLE 4.0 beacon and pedometer technologies. The study designed a system to direct user using a smartphone application working on an android platform by creating map and providing direction for user to navigate from one location to another indoor. The results show that the system can guide user accurately, be used effectively, and does not require additional components. The combination of the three technologies has improved the performance of the system. User may choose the strongest of available signals supported by the system. The study has achieved satisfactory results by 
combining three approaches to simplify the system workflow so it can be used seamlessly by user. The positioning system based on Wi-Fi, BLE beacon and pedometer can be used in a multi-user environment and in different situations. The system was proven more accurate in pinpointing location indoors than the existing systems.

The indoor localization system presented in this study also provides highlighted path according to the user current location and destination, in addition to being fast responding, secure and robust. These qualities contribute to the authenticity of the system, whereby combination of the three indoor technologies and correct recording prioritize user's interaction in the application. User can see the option for additional points on the map, indoor tracking and data graphs; user's current location and destination are indicated and recorded in the system as they move from outdoor to indoor space.

Future research is needed to compare the performance of the proposed system with other indoor positioning systems. It is also worthwhile to try to integrate the system with existing outdoor positioning systems to create a seamless environment and better performance using smartphones, tablets and other multipurpose devices.

\section{Conflicts of Interest}

The authors declare no conflict of interest.

\section{Author Contributions}

The research was conducted by Khamla Nonalinsavath, Lukito Edi Nugroho, Widyawan and Kazuhiko Hamamoto; this work was carried out as a part of my Ph.D. dissertation; Khamla NonAlinsavath wrote the paper, prepared all related works and collected data from other authors. Lukito Edi Nugroho, Widyawan and Kazuhiko Hamamoto analyzed, revised the data and checked all content of this research; They also acted as supervisors and guided of this research work; all authors had approved the final version.

\section{Acknowledgments}

I would like to express my deepest gratitude to my advisors, and colleagues for their technical and/or material support and assistance. Universitas Gadjah Mada and Tokai University are acknowledged for their support and facilitation in this research. I would like to thank the AUN-SEED/Net (ASEAN University Network/Southeast Asia Engineering Education Development Network) Program, JICA
(Japan International Cooperation Agency) Project for the financial support.

\section{References}

[1] Y. Lee and K. Lien, "Location Based Enabled Context Awareness Information Service", In: Proc. of 2009 International Conference on New Trends in Information and Service Science, pp. 944-947, 2009. doi: 10.1109/NISS.2009.160

[2] K. Lee, J. Lee, and M.-P. Kwan, "Locationbased service using ontology-based semantic queries: A study with a focus on indoor activities in a university context", Comput. Environ. Urban Syst., Vol. 62, pp. 41-52, 2017. doi: https://doi.org/10.1016/j.compenvurbsys.2016.1 0.009

[3] C. Wu, Q. Mu, Z. Zhang, Y. Jin, Z. Wang, and G. Shi, "Indoor positioning system based on inertial MEMS sensors: Design and realization", In: Proc. of 2016 IEEE International Conference on Cyber Technology in Automation, Control, and Intelligent Systems (CYBER), pp. 370-375, 2016. doi: 10.1109/CYBER.2016. 7574852

[4] T. H. Nyein and A. M. Yi, "Location Based Personal Task Reminder System Using GPS Technology", In: Proc. of Big Data Analysis and Deep Learning Applications, pp. 224-230, 2019.

[5] W. K. Zegeye, S. B. Amsalu, Y. Astatke, and F. Moazzami, "WiFi RSS fingerprinting indoor localization for mobile devices", In: Proc. of 2016 IEEE 7th Annual Ubiquitous Computing, Electronics Mobile Communication Conference (UEMCON), pp. 1-6, 2016. doi: 10.1109/ UEMCON.2016.7777834

[6] A. A. Kalbandhe and S. C. Patil, "Indoor Positioning System using Bluetooth Low Energy", In: Proc. of 2016 International Conference on Computing, Analytics and Security Trends (CAST), pp. 451-455, 2016. doi: 10.1109/CAST.2016.7915011

[7] S. S. Saab and Z. S. Nakad, "A Standalone RFID Indoor Positioning System Using Passive Tags", IEEE Trans. Ind. Electron., Vol. 58, No. 5, pp. 1961-1970, 2011. doi: 10.1109/TIE.2010. 2055774

[8] P. V. Thanh, A. N. Thi, Q. T. T. Thuy, D. C. T. Phuong, V. H. Mau, and D. Tran, "A novel step counter supporting for indoor positioning based on inertial measurement unit", In: Proc. of 2017 7 th International Conference on Integrated Circuits, Design, and Verification (ICDV), pp. 69-74, 2017. doi: 10.1109/ICDV.2017.8188641 
[9] M. Uradzinski, H. Guo, X. Liu, and M. Yu, "Advanced Indoor Positioning Using Zigbee Wireless Technology", Wirel. Pers. Commun., Vol. 97, No. 4, pp. 6509-6518, 2017. doi: 10.1007/s11277-017-4852-5

[10] Y.-C. Pu and P.-C. You, "Indoor positioning system based on BLE location fingerprinting with classification approach", Appl. Math. Model., Vol. 62, pp. 654-663, 2018, doi: https://doi.org/10.1016/j.apm.2018.06.031.

[11] A. Basiri, E. Lohan, T. Moore, A. Winstanley, and P. Peltola, "Indoor location based services challenges, requirements and usability of current solutions", Comput. Sci. Rev., Vol. 24, pp. 1-12, 2017. doi: 10.1016/j.cosrev.2017.03.002

[12] S. Adler, S. Schmitt, K. Wolter, and M. Kyas, "A survey of experimental evaluation in indoor localization research", In: Proc. of 2015 International Conference on Indoor Positioning and Indoor Navigation (IPIN), pp. 1-10, 2015. doi: 10.1109/IPIN.2015.7346749

[13] S. Alletto, R. Cucchiara, G. Fiore, L. Mainetti, V. Mighali, L. Patrono, and G. Serra, "An Indoor Location-Aware System for an IoT-Based Smart Museum", IEEE Internet Things J., Vol. 3, pp. 244-253, 2016.

[14] R. Wilfinger, T. Moder, M. Wieser, and B. Grosswindhager, "Indoor position determination using location fingerprinting and vehicle sensor data", In: Proc. of 2016 European Navigation Conference (ENC), pp. 1-9, 2016. doi: 10.1109/EURONAV.2016.7530561

[15] P. K. Binu, R. A. Krishnan, and A. P. Kumar, "An efficient indoor location tracking and navigation system using simple magnetic map matching", In: Proc. of 2016 IEEE International Conference on Computational Intelligence and Computing Research (ICCIC), pp. 1-7, 2016. doi: 10.1109/ICCIC.2016.7919537

[16] W. Jiang, Y. Li, C. Rizos, B. Cai, and W. Shangguan, "Seamless Indoor-Outdoor Navigation based on GNSS, INS and Terrestrial Ranging Techniques", J. Navig., Vol. 70, No. 6, pp. 1183-1204, 2017.

[17] H.-H. Liu and C. Liu, "Implementation of Wi-Fi Signal Sampling on an Android Smartphone for Indoor Positioning Systems", Sensors, Vol. 18, No. 1, p. 3, 2017. doi: 10.3390/s18010003

[18] S. Safei, "INDOOR POSITION DETECTION USING WIFI AND TRILATERATION TECHNIQUE", Int. Conf. Inform. Appl. ICIA2012, pp. 362-366, 2012.

[19] R. F. Brena, J. P. García-Vázquez, C. E. GalvánTejada, D. Muñoz-Rodriguez, C. VargasRosales, and J. Fangmeyer, "Evolution of Indoor
Positioning Technologies: A Survey", J. Sens., Vol. 2017, p. 2630413, 2017. doi: 10.1155/2017/ 2630413.

[20] O. J. Woodman and R. K. Harle, "Concurrent scheduling in the Active Bat location system", In: Proc. of 2010 8th IEEE International Conference on Pervasive Computing and Communications Workshops (PERCOM Workshops), pp. 431-437, 2010. doi: 10.1109/ PERCOMW.2010.5470631.

[21] "Mall of America," Wikipedia. Mar. 24, 2020, Accessed: Apr. 15, 2020. [Online]. Available: https://en.wikipedia.org/w/index.php?title=Mall _of_America\&oldid=947055256 